\title{
THE PERFECT LOCAL $T b$ THEOREM AND TWISTED MARTINGALE TRANSFORMS
}

\author{
MICHAEL T. LACEY AND ANTTI V. VÄHÄKANGAS
}

(Communicated by Alexander Iosevich)

\begin{abstract}
A local $T b$ Theorem provides a flexible framework for proving the boundedness of a Calderón-Zygmund operator $T$. One needs only boundedness of the operator $T$ on systems of locally pseudo-accretive functions $\left\{b_{Q}\right\}$, indexed by cubes. We give a new proof of this theorem in the setting of perfect (dyadic) models of Calderón-Zygmund operators, imposing integrability conditions on the $b_{Q}$ functions that are the weakest possible. The proof is a simple direct argument, based upon an inequality for transforms of so-called twisted martingale differences, which has been noted by Auscher-Routin.
\end{abstract}

\section{INTRODUCTION}

An operator $T$ is said to be a perfect Calderón-Zygmund operator if it satisfies the following conditions. There is a kernel $K(x, y)$ so that

$$
\langle T f, g\rangle=\int_{\mathbb{R}^{n}} \int_{\mathbb{R}^{n}} K(x, y) f(y) g(x) d y d x
$$

for all $f, g$ that are smooth, compactly supported, and the closure of the supports of $f$ and $g$ do not intersect. The kernel $K(x, y)$ is assumed to satisfy the size condition

$$
|K(x, y)| \leq \frac{1}{|x-y|^{n}}
$$

and it satisfies the following strong smoothness condition. For any two disjoint dyadic cubes $P, Q, K(x, y)$ is constant on $P \times Q$. The implication of this property, used repeatedly, is this: If $f$ is supported on $P$ and $g$ is supported on $Q$, and at least one of $f$ and $g$ have integral zero, then $\langle T f, g\rangle=0$.

We are interested in bounded Calderón-Zygmund operators, so we set $\mathbf{T}$ to be the norm of $T$ on $L^{2}\left(\mathbb{R}^{n}\right)$; namely, $\mathbf{T}$ is the best constant in the inequality

$$
|\langle T f, g\rangle| \leq \mathbf{T}\|f\|_{2}\|g\|_{2} \text {. }
$$

It is well known that this inequality extends to the form $|\langle T f, g\rangle| \lesssim \mathbf{T}\|f\|_{p}\|g\|_{p^{\prime}}$, where $1<p<\infty$ and $1 / p+1 / p^{\prime}=1$.

The celebrated $T 1$ Theorem of David and Journé [10] gives a beautiful characterization of the bounded Calderón-Zygmund operators. It was the powerful observation of Michael Christ [8] that a weakening of the $T 1$ Theorem to a so-called $T b$

Received by the editors April 29, 2012 and, in revised form, May 14, 2012 and June 19, 2012. 2010 Mathematics Subject Classification. Primary 42B20.

This research was supported in part by grant NSF-DMS 0968499 and a grant from the Simons Foundation (\#229596) to the first author.

The second author was supported by the School of Mathematics, Georgia Institute of Technology, and by the Finnish Academy of Science and Letters, Vilho, Yrjö and Kalle Väisälä Foundation. 
formulation can have wide-ranging implications. Christ himself addressed analytic capacity, and it was this perspective that was crucial to the solution of the Kato square root problem [2,12]. Our focus is on the local $T b$, in the dyadic model, as promoted in [1]. This is the usual definition of systems of accretive functions.

Definition 1.2. Fix $1<p<\infty$. A collection of functions $\left\{b_{Q}: Q \in \mathcal{D}\right\}$ is called a system of $p$-accretive functions with constant $1<\mathbf{A}$ if the following conditions hold for each dyadic cube $Q \in \mathcal{D}$ :

(1) $b_{Q}$ is supported on $Q$ and $\int_{Q} b_{Q}(x) d x=|Q|$.

(2) $\left\|b_{Q}\right\|_{p} \leq \mathbf{A}|Q|^{1 / p}$.

In the theorem below $p_{1}, p_{2}$ are not related by duality; for instance it is allowed that $1<p_{1}, p_{2}<2$.

Theorem 1.3. For fixed constants $\mathbf{A}$ and $\mathbf{T}_{\mathrm{loc}}$, this holds. Suppose that $T$ is a perfect dyadic Calderón-Zygmund operator and, for $1<p_{1}, p_{2}<\infty$, there are systems $\left\{b_{Q}^{j}\right\}$ of $p_{j}$-accretive functions with constant $\mathbf{A}$, so that

$$
\int_{Q}\left|T b_{Q}^{1}\right|^{p_{2}^{\prime}} d x \leq \mathbf{T}_{\mathrm{loc}}^{p_{2}^{\prime}}|Q|, \quad \int_{Q}\left|T^{*} b_{Q}^{2}\right|^{p_{1}^{\prime}} d x \leq \mathbf{T}_{\mathrm{loc}}^{p_{1}^{\prime}}|Q| .
$$

Then, $T$ extends to a bounded operator on $L^{2}$, and moreover, $\mathbf{T} \lesssim_{\mathbf{A}, p_{1}, p_{2}} 1+\mathbf{T}_{\text {loc }}$.

This is a known result [1, Theorem 6.8]. Auscher and Routin [3, Section 8] have recently devised a proof closely related to this one.

Martingale transform inequalities for twisted differences play a central role. These inequalities have also been used by Auscher-Routin [3, Section 5]. Our direct proof of Theorem 1.3 proceeds by standard reductions, the construction of stopping cubes from the local $T b$ hypotheses, and a brief additional argument. A highlight in the present paper is a simple appeal to the local $T b$ hypothesis and the martingale transform inequality. Compare to [3, Estimate for $\left.\left\langle f, V_{1,2} g\right\rangle\right]$.

Relevant history and indications of the utility of $T b$ theorems can be found in surveys by S. Hofmann [13, 14]. See in particular [13, §3.3.1], where an extension of Theorem 1.3 to the continuous case is specifically mentioned. The perfect case is of course very special. Still the argument in [1] has been influential, although the task of lifting the proof therein to the continuous case has not proven to be easy.

Auscher and Yang 4 succeeded in extending the theorem above to the continuous case, with the duality assumption on $p_{1}$ and $p_{2}$, but the argument is an indirect reduction to the perfect case. This is less desirable, due to the interest in local $T b$ theorems in more general settings, such as the setting of homogeneous spaces, as in Auscher and Routin [3]. The latter paper follows an adaptation of the socalled Beylkin-Coifman-Rokhlin (BCR) algorithm [5, 11], as well as the martingale transform inequalities, and it also addresses the case of $1 / p_{1}+1 / p_{2}>1$, but additional hypotheses are needed. These hypotheses have very recently been removed by T. Hytönen and F. Nazarov [17]. By applying perturbation techniques for both the operator and the accretive functions, they obtain a variant of Theorem 1.3 for all exponents $p_{1}, p_{2} \in(1, \infty)$, and in the continuous case.

One can also consult Hytönen-Martikainen [15,16] for another general approach to the local $T b$ Theorem in non-homogeneous and upper doubling settings, although in that setting duality is imposed. A local $T b$ Theorem in a vector-valued setting, with strong conditions on accretive functions, is considered in [18. Salamone [24] also studies the dyadic $T b$ Theorem. 
Notation. For any cube $Q,\langle f\rangle_{Q}:=|Q|^{-1} \int_{Q} f d x$, and $\ell Q=|Q|^{1 / n}$ is the side length of the cube. $A \lesssim B$ means that $A \leq C \cdot B$, where $C$ is an unspecified constant independent of $A$ and $B$. We will work with different subfamilies of the dyadic grid $\mathcal{D}$ and need some notation. Given $Q \in \mathcal{D}$, we denote by $\operatorname{ch}(Q)$ the $2^{n}$ dyadic children of $Q$. Given $\mathcal{S} \subset \mathcal{D}$, we can refer to the $\mathcal{S}$-children of $S \in \mathcal{S}$ : the maximal elements $S^{\prime}$ of $\mathcal{S}$ that are strictly contained in $S$. This collection is denoted as $\operatorname{ch}_{\mathcal{S}}(S)$. For a cube $Q \in \mathcal{D}$, contained in a cube in $\mathcal{S}$, we take $\pi_{\mathcal{S}} Q$ to be the $\mathcal{S}$-parent of $Q$ : the smallest cube in $\mathcal{S}$ that contains $Q$. The dyadic parent is $\pi Q$.

\section{The Martingale transform inequality}

The classical martingale transform inequality is this: For all constants satisfying $\left|\varepsilon_{Q}\right| \leq 1$

$$
\left\|\sum_{Q \in \mathcal{D}} \varepsilon_{Q} \sum_{Q^{\prime} \in \operatorname{ch}(Q)}\left\{\langle f\rangle_{Q^{\prime}}-\langle f\rangle_{Q}\right\} \mathbf{1}_{Q^{\prime}}\right\|_{q} \lesssim\|f\|_{q}, \quad 1<q<\infty
$$

see e.g. 6, 7. A variant is stated in Theorem 2.3, and it is essential to the subsequent arguments. This section can be read independently of the rest of the paper.

Fix a function $b$ supported on a dyadic cube $S_{0}$, satisfying $\int b d x=\left|S_{0}\right|$, and $\|b\|_{p} \leq \mathbf{A}\left|S_{0}\right|^{1 / p}$, where $1<p<\infty$ is fixed. Let $\mathcal{T}^{\prime}$ be the maximal dyadic cubes $T \subset S_{0}$ which meet either one of the following conditions with $\delta \in(0,1)$ :

$$
\left|\int_{T} b d x\right| \leq \delta|T| \quad \text { or } \quad \int_{T}|b|^{p} d x \geq \delta^{-1} \mathbf{A}^{p}|T| .
$$

We will consider a fixed but arbitrary family $\mathcal{T}$ of disjoint dyadic cubes in $S_{0}$, the 'terminal cubes', and our estimates are not allowed to depend upon this family. Moreover, we require that $T^{\prime} \subset T \in \mathcal{T}$ if $T^{\prime} \in \mathcal{T}^{\prime}$. For each terminal cube $T$, we have a function $b_{T}$ supported on $T$ and satisfying $\int b_{T} d x=|T|$ and $\left\|b_{T}\right\|_{p} \leq \mathbf{A}|T|^{1 / p}$.

Let $\mathcal{Q}$ be all dyadic cubes contained in $S_{0}$, but not contained in any $T \in \mathcal{T}$. Define

$$
\Delta_{Q} f:=\sum_{Q^{\prime} \in \operatorname{ch}(Q)}\left\{\frac{\langle f\rangle_{Q^{\prime}}}{\left\langle b_{Q^{\prime}}\right\rangle_{Q^{\prime}}} b_{Q^{\prime}}-\frac{\langle f\rangle_{Q}}{\langle b\rangle_{Q}} b\right\} \mathbf{1}_{Q^{\prime}}, \quad Q \in \mathcal{Q},
$$

where we set $b_{Q^{\prime}}=b$ if $Q^{\prime} \notin \mathcal{T}$, and otherwise, $b_{Q^{\prime}}$ is defined as above. We refer to $\Delta_{Q} f$ as the twisted martingale difference.

Theorem 2.3. This inequality holds for all selections of constants $\left|\varepsilon_{Q}\right| \leq 1$ :

$$
\left\|\sum_{Q \in \mathcal{Q}} \varepsilon_{Q} \Delta_{Q} f\right\|_{p} \lesssim\|f\|_{p}
$$

where $1<p<\infty$ is the exponent associated with the function $b$.

This theorem and Theorem 2.6 below are contained in [3, Lemma 5.3]. Also, a randomized version of the theorem above in a vector-valued context is proven in [18, Section 4]. We give a somewhat different proof, in the spirit of completeness, since we view these inequalities as fundamental to the $T b$ theorems.

We need the following preparation. In the sum below, we do not sum over the children which are terminal cubes, and we do not multiply by the $b$ functions, and 
so we refer to these as the half-twisted differences:

$$
D_{Q} f:=\sum_{Q^{\prime} \in \operatorname{ch}(Q) \backslash \mathcal{T}}\left\{\frac{\langle f\rangle_{Q^{\prime}}}{\langle b\rangle_{Q^{\prime}}}-\frac{\langle f\rangle_{Q}}{\langle b\rangle_{Q}}\right\} \mathbf{1}_{Q^{\prime}}, \quad Q \in \mathcal{Q} .
$$

The following universal estimate holds in Lebesgue measure.

Theorem 2.6. The following inequalities hold for all selections of constants $\left|\varepsilon_{Q}\right| \leq$ 1:

$$
\left\|\sum_{Q \in \mathcal{Q}} \varepsilon_{Q} D_{Q} f\right\|_{q} \lesssim\|f\|_{q}, \quad 1<q<\infty
$$

Proof. It is important to note that this operator is, in fact, a constant multiple of a perfect Calderón-Zygmund operator. It therefore suffices to verify the conditions of the $T 1$ Theorem, but this is not convenient to do directly. Instead, we write the operator as a sum of three perfect Calderón-Zygmund operators and consider each of them separately.

For cube $Q \in \mathcal{Q}$ with child $Q^{\prime} \notin \mathcal{T}$, we write

$$
\begin{aligned}
\frac{\langle f\rangle_{Q^{\prime}}}{\langle b\rangle_{Q^{\prime}}}-\frac{\langle f\rangle_{Q}}{\langle b\rangle_{Q}}= & \left\{\frac{\langle f\rangle_{Q^{\prime}}}{\langle b\rangle_{Q}}-\frac{\langle f\rangle_{Q}}{\langle b\rangle_{Q}}\right\}+\left\{\frac{\langle f\rangle_{Q^{\prime}}}{\langle b\rangle_{Q^{\prime}}}-\frac{\langle f\rangle_{Q^{\prime}}}{\langle b\rangle_{Q}}\right\} \\
= & \left\{\frac{\langle f\rangle_{Q^{\prime}}}{\langle b\rangle_{Q}}-\frac{\langle f\rangle_{Q}}{\langle b\rangle_{Q}}\right\}+\left\{\langle b\rangle_{Q}-\langle b\rangle_{Q^{\prime}}\right\} \frac{\langle f\rangle_{Q^{\prime}}}{\langle b\rangle_{Q^{\prime}}\langle b\rangle_{Q}} \\
= & \left\{\frac{\langle f\rangle_{Q^{\prime}}}{\langle b\rangle_{Q}}-\frac{\langle f\rangle_{Q}}{\langle b\rangle_{Q}}\right\} \\
& +\left\{\langle b\rangle_{Q}-\langle b\rangle_{Q^{\prime}}\right\} \frac{\langle f\rangle_{Q^{\prime}}}{\langle b\rangle_{Q}^{2}} \\
& +\left\{\langle b\rangle_{Q}-\langle b\rangle_{Q^{\prime}}\right\}^{2} \frac{\langle f\rangle_{Q^{\prime}}}{\langle b\rangle_{Q^{\prime}}\langle b\rangle_{Q^{2}}{ }^{2}} .
\end{aligned}
$$

This gives us three sums to bound. Keep in mind that the averages of $b$ that occur are bounded from above and below by the failure of (2.2). In the first two expressions, the denominator is only a function of $Q$, while in the third, it depends upon the child $Q^{\prime}$, with however the square on the difference on $b$. The first term gives rise to a classical martingale difference on $f$, the second a martingale difference on $b$, and the third, a square function of a martingale difference on $b$.

The desired estimate for the sum with term (2.10) follows from the following observation:

$$
\left\|\sum_{Q \in \mathcal{Q}} \varepsilon_{Q} \sum_{Q^{\prime} \in \operatorname{ch}(Q) \backslash \mathcal{T}}\left\{\langle f\rangle_{Q^{\prime}}-\langle f\rangle_{Q}\right\} \mathbf{1}_{Q^{\prime}}\right\|_{q} \lesssim\|f\|_{q}, \quad 1<q<\infty .
$$

Indeed, this is a consequence of the classical martingale transform inequality (2.1) and maximal function estimates in the disjoint family of missing terminal cubes.

An estimate for the sum associated with term (2.11) is clearly a consequence of the inequality

$$
\left\|\sum_{Q \in \mathcal{Q}} \varepsilon_{Q} \sum_{Q^{\prime} \in \operatorname{ch}(Q) \backslash \mathcal{T}}\left\{\langle b\rangle_{Q^{\prime}}-\langle b\rangle_{Q}\right\}\langle f\rangle_{Q^{\prime}} \mathbf{1}_{Q^{\prime}}\right\|_{q} \lesssim \mathbf{A}\|f\|_{q}, \quad 1<q<\infty .
$$

Denote the linear operator on the left hand side by $\Pi$. After normalizing with a constant $c_{n, \delta} \mathbf{A}^{-1}$, we are looking for $L^{q}$-norm estimates for a symmetric perfect 
Calderón-Zygmund operator - recall this definition from the Introduction. We appeal to the local $T 1$ Theorem for such operators (see e.g. [1, Corollary 6.3] or $[13,25]$ ), testing the $L^{1}$-norm of $\Pi \mathbf{1}_{F}$ for all dyadic cubes $F$. Indeed, it suffices to verify the inequality

$$
\left\|\Pi \mathbf{1}_{F}\right\|_{L^{1}(F)} \lesssim|F|, \quad F \in \mathcal{D} .
$$

By Hölder, inequality $\left\|\Pi \mathbf{1}_{F}\right\|_{L^{p}} \lesssim|F|^{1 / p}$ suffices for (2.15). Let us write

$$
\Pi \mathbf{1}_{F}=\left\{\sum_{\substack{Q \in \mathcal{Q} \\ Q \supsetneq F}}+\sum_{\substack{Q \in \mathcal{Q} \\ Q \subset F}}\right\} \varepsilon_{Q} \sum_{Q^{\prime} \in \operatorname{ch}(Q) \backslash \mathcal{T}}\left\{\langle b\rangle_{Q^{\prime}}-\langle b\rangle_{Q}\right\}\left\langle\mathbf{1}_{F}\right\rangle_{Q^{\prime}} \mathbf{1}_{Q^{\prime}}
$$

Using Minkowski inequality and the trivial estimate $\left|\left\langle\mathbf{1}_{F}\right\rangle_{Q^{\prime}}\right| \leq|F|\left|Q^{\prime}\right|^{-1}$, we find that the $L^{p}$-norm of the first series is bounded by $c_{n} \delta^{-1 / p} \mathbf{A}|F|^{1 / p}$. Concerning the second series, we can clearly assume that $Q \subset F$ for some $Q \in \mathcal{Q}$. Let us denote by $R$ the maximal cube in $\mathcal{Q}$, contained in $F$. Assuming $\mathcal{Q} \ni Q \subset F$ and $Q^{\prime}$ is a child of $Q$, then $\left\langle\mathbf{1}_{F}\right\rangle_{Q^{\prime}}=1,\langle b\rangle_{Q^{\prime}}=\left\langle b \mathbf{1}_{R}\right\rangle_{Q^{\prime}}$, and likewise $\langle b\rangle_{Q}=\left\langle b \mathbf{1}_{R}\right\rangle_{Q}$. By inequality (2.13), setting $\varepsilon_{Q}=0$ if $Q \supsetneq F$, the $L^{p}$-norm of the second series in (2.16) is bounded by $\left\|b \mathbf{1}_{R}\right\|_{p}$ which, in turn, is bounded by $\delta^{-1 / p} \mathbf{A}|F|^{1 / p}$. This concludes the proof of inequality (2.15), and, as a consequence, we obtain inequality (2.14).

It remains to estimate the sum associated with term (2.12). Namely, we need the following inequality:

$$
\left\|\sum_{Q \in \mathcal{Q}} \varepsilon_{Q} \sum_{Q^{\prime} \in \operatorname{ch}(Q) \backslash \mathcal{T}}\left\{\langle b\rangle_{Q^{\prime}}-\langle b\rangle_{Q}\right\}^{2} \frac{\langle f\rangle_{Q^{\prime}}}{\langle b\rangle_{Q^{\prime}}\langle b\rangle_{Q}^{2}} \mathbf{1}_{Q^{\prime}}\right\|_{q} \lesssim \mathbf{A}\|f\|_{q}, \quad 1<q<\infty .
$$

Denote the linear operator on the left hand side by $\amalg$. Again, after a normalization by $c_{n, \delta} \mathbf{A}^{-2}$, we are looking for $L^{q}$ estimates of the perfect Calderón-Zygmund operator. By symmetry of $\amalg$, it suffices to verify that

$$
\left\|\amalg \mathbf{1}_{F}\right\|_{L^{1}(F)} \lesssim|F|, \quad F \in \mathcal{D} .
$$

In order to do this, we split the series defining $\amalg \mathbf{1}_{F}$ into two parts as above, one with cubes $Q \supsetneq F$ and the other with cubes $Q \subset F$. Reasoning as above, we find that the $L^{2}$-norm of the first series is bounded by $c_{n, \delta} \mathbf{A}^{2}|F|^{1 / 2}$, and the corresponding $L^{1}(F)$-norm estimate follows. The second series to estimate is

$$
\left\|\sum_{\substack{Q \in \mathcal{Q} \\ Q \subset F}} \varepsilon_{Q} \sum_{Q^{\prime} \in \operatorname{ch}(Q) \backslash \mathcal{T}}\left\{\langle b\rangle_{Q^{\prime}}-\langle b\rangle_{Q}\right\}^{2} \frac{\left\langle\mathbf{1}_{F}\right\rangle_{Q^{\prime}}}{\langle b\rangle_{Q^{\prime}}\langle b\rangle_{Q}^{2}} \mathbf{1}_{Q^{\prime}}\right\|_{1} .
$$

Using the facts that $\left\langle\mathbf{1}_{F}\right\rangle_{Q^{\prime}}=1$ if $Q \subset F$ and $Q^{\prime}$ is a child of $Q$, we obtain the upper bound

$$
c_{\delta} \sum_{Q \in \mathcal{Q}} \sum_{Q^{\prime} \in \operatorname{ch}(Q) \backslash \mathcal{T}} \int_{\mathbf{R}^{n}}\left|\left\{\langle b\rangle_{Q^{\prime}}-\langle b\rangle_{Q}\right\}\left\langle\mathbf{1}_{F}\right\rangle_{Q^{\prime}} \mathbf{1}_{Q^{\prime}}(x)\right|^{2} d x,
$$

where we have also relaxed the summation condition using positivity of the summands. This upper bound, in turn, is bounded by a constant multiple of $\mathbf{A}^{2}\left\|\mathbf{1}_{F}\right\|_{2}^{2}=$ $\mathbf{A}^{2}|F|$, a consequence of a square function estimate arising from randomization of inequality (2.14) with $q=2$ by taking $\varepsilon_{Q}$ 's to be Rademacher random variables. This completes the proof of inequality (2.18) and, consequently, the proof of the theorem. 
We have the following easier proposition.

Proposition 2.19. The following inequality holds for all selections of constants $\left|\varepsilon_{Q}\right| \leq 1$ :

$$
\left\|\sum_{T \in \mathcal{T}} \varepsilon_{\pi T}\langle f\rangle_{T} \mathbf{1}_{T}\right\|_{q}+\left\|\sum_{T \in \mathcal{T}} \varepsilon_{\pi T} \frac{\langle f\rangle_{\pi T}}{\langle b\rangle_{\pi T}} \mathbf{1}_{T}\right\|_{q} \lesssim\|f\|_{q}, \quad 1<q<\infty .
$$

Proof. By disjointness of terminal cubes and the estimate $\left|\langle b\rangle_{\pi T}\right| \geq \delta$ for $T \in \mathcal{T}$,

$$
\begin{aligned}
\left\|\sum_{T \in \mathcal{T}} \varepsilon_{\pi T} \frac{\langle f\rangle_{\pi T}}{\langle b\rangle_{\pi T}} \mathbf{1}_{T}\right\|_{q} & \leq \delta^{-1}\left(\sum_{T \in \mathcal{T}} \int_{\mathbf{R}^{n}}\left|\langle f\rangle_{\pi T} \mathbf{1}_{T}(x)\right|^{q} d x\right)^{1 / q} \\
& \lesssim \delta^{-1}\|M f\|_{q} \lesssim \delta^{-1}\|f\|_{q} .
\end{aligned}
$$

The other term is estimated in a similar manner.

Proof of Theorem 2.3. Let us set $B f:=\sum_{Q \in \mathcal{Q}} \varepsilon_{Q} D_{Q} f$, and observe that

$$
\sum_{Q \in \mathcal{Q}} \varepsilon_{Q} \Delta_{Q} f=B f \cdot b+\sum_{T \in \mathcal{T}} \varepsilon_{\pi T}\langle f\rangle_{T} \mathbf{1}_{T} \cdot \sum_{T \in \mathcal{T}} b_{T}-\sum_{T \in \mathcal{T}} \varepsilon_{\pi T} \frac{\langle f\rangle_{\pi T}}{\langle b\rangle_{\pi T}} \mathbf{1}_{T} \cdot b .
$$

Consider the events $E_{\lambda}:=\{|B f| \geq \lambda\} \subset S_{0}$, where $\lambda>0$. Let $S_{\mathcal{T}} \subset S_{0}$ be the union of terminal cubes $T \in \mathcal{T}$. By construction of $\mathcal{T}$ and the Lebesgue differentiation theorem, we have $|b(x)| \leq \delta^{-1 / p} \mathbf{A}$ for almost every $x \in S_{0} \backslash S_{\mathcal{T}}$. Hence,

$$
\int_{E_{\lambda} \backslash S_{\mathcal{T}}}|b|^{p} d x \leq \delta^{-1} \mathbf{A}^{p}\left|E_{\lambda} \backslash S_{\mathcal{T}}\right|
$$

Observe that $B f$ is constant on terminal cubes. Let us denote $\mathcal{T}_{\lambda}:=\{T \in \mathcal{T}$ : $|B f| \geq \lambda$ on $T\}$. Since $\pi T \in \mathcal{Q}$ for each terminal cube $T$,

$$
\begin{aligned}
\int_{E_{\lambda} \cap S_{\mathcal{T}}}|b|^{p} d x & =\sum_{T \in \mathcal{T}_{\lambda}}|T|\left(\frac{1}{|T|} \int_{T}|b|^{p} d x\right) \\
& \leq 2^{n} \delta^{-1} \mathbf{A}^{p} \sum_{T \in \mathcal{T}_{\lambda}}|T| \leq 2^{n} \delta^{-1} \mathbf{A}^{p}\left|E_{\lambda} \cap S_{\mathcal{T}}\right|
\end{aligned}
$$

It follows that we can compare Lebesgue measure estimates and estimates with respect to measure $|b|^{p} d x$, namely,

$$
\int_{E_{\lambda}}|b|^{p} d x \leq 2^{n} \delta^{-1} \mathbf{A}^{p}\left|E_{\lambda}\right| .
$$

Therefore, by a standard formula and the Lebesgue measure estimates of Theorem 2.6.

$$
\int_{S_{0}}|B f|^{p}|b|^{p} d x=p \int_{0}^{\infty} \lambda^{p-1} \int_{E_{\lambda}}|b|^{p} d x d \lambda \lesssim \mathbf{A}^{3 p}\|f\|_{p}^{p}
$$

The $L^{p}$-norms of the two remaining quantities on the right hand side of (2.20) are estimated in a similar manner by using Proposition 2.19 and measures $\sum_{T \in \mathcal{T}}\left|b_{T}\right|^{p} d x$ and $|b(x)|^{p} d x$ instead. 


\section{THE CORONA}

This is the construction of stopping cubes: for a fixed $Q_{0} \in \mathcal{D}$, families $\mathcal{S}_{1}, \mathcal{S}_{2} \subset$ $\mathcal{D}$ are defined as follows. Take the cube $Q_{0}$ in $\mathcal{S}_{1}$. In the inductive stage, if $S \in \mathcal{S}_{1}$, take as members of $\mathcal{S}_{1}$ those maximal dyadic descendants $Q$ which meet any one of these several conditions:

(1) $\left|\int_{Q} b_{S}^{1}\right| \leq \delta|Q|$.

(2) $\int_{Q}\left|b_{S}^{1}\right|^{p_{1}} \geq \delta^{-1} \mathbf{A}^{p_{1}}|Q|$.

(3) $\int_{Q}\left|T b_{S}^{1}\right|^{p_{2}^{\prime}} \geq \delta^{-1} \mathbf{T}_{\mathrm{loc}}^{p_{2}^{\prime}}|Q|$.

A stopping tree $\mathcal{S}_{2}$ is then constructed analogously, but using functions $\left\{b_{S}^{2}\right\}_{S \in \mathcal{D}}$, and exponents $p_{2}$ and $p_{1}^{\prime}$ in conditions (2) and (3), respectively. Furthermore, in (3) we use $T^{*}$ instead of $T$.

If $\delta>0$ is chosen sufficiently small in the construction above, then there is a constant $\tau \in(0,1)$ such that

$$
\sum_{S^{\prime} \in \operatorname{ch}_{\mathcal{S}_{j}}(S)}\left|S^{\prime}\right| \leq \tau|S|, \quad S \in \mathcal{S}_{j}, \quad j \in\{1,2\} .
$$

Here both $\tau$ and $\delta$ depend on A. It follows from construction and inequality (3.1) that $\mathcal{S}:=\mathcal{S}_{1} \cup \mathcal{S}_{2}$ is a Carleson family of cubes. Namely, there holds

$$
\sum_{S \in \mathcal{S}: S \subset Q}|S| \lesssim_{\tau}|Q|, \quad \mathcal{D} \ni Q \subset Q_{0}
$$

In the sequel, we suppose that $\delta$ is chosen as above.

Remark 3.3. With $\mathcal{S}$ so constructed, many subsequent inequalities have constants that depend upon the values of $\delta$ and $\mathbf{A}$. The dependence is not straightforward, and we do not attempt to track it. Frequently, this dependence is even suppressed in the notation, so that in many parts of the argument relative to $\mathcal{S}$, the symbol ' $\lesssim$ ' should be read as 'the unspecified implied constant depends upon dimension, $\tau, \delta$ and $\mathbf{A}$, but is otherwise absolute'. Dependencies on other parameters are indicated by subscripts; e.g. $\lesssim_{p} 1$ means $\lesssim c_{p}$, where $c_{p}$ depends on $p$ only.

Following [9], we define the $b$-adapted conditional expectations and martingale differences, associated with a dyadic cube $Q \subset Q_{0}$, by

$$
E_{Q}^{j} h:=\frac{\langle h\rangle_{Q}}{\left\langle b_{\pi_{\mathcal{S}_{j} Q}}^{j}\right\rangle_{Q}} b_{\pi_{\mathcal{S}_{j} Q}}^{j} \mathbf{1}_{Q}, \quad \Delta_{Q}^{j} h:=\sum_{Q^{\prime} \in \operatorname{ch}(Q)}\left\{E_{Q^{\prime}}^{j} h-E_{Q^{j}}^{j} h\right\} \mathbf{1}_{Q^{\prime}} .
$$

Observe that the functions $\Delta_{Q}^{j} h$ have mean zero in $\mathbf{R}^{n}$, and they are supported on the cube $Q$.

The following lemma is proven in the same way as Lemma 3.5 in [16], with obvious modifications.

Lemma 3.5. Let $h \in L^{p_{j}}\left(\mathbf{R}^{n}\right), j \in\{1,2\}$. Then, there holds pointwise and in $L^{p_{j}}\left(\mathbf{R}^{n}\right)$,

$$
h \mathbf{1}_{S}=E_{S}^{j} h+\sum_{Q: Q \subset S} \Delta_{Q}^{j} h, \quad S \subset Q_{0} .
$$

We will suppress the notation by denoting $E_{Q}^{j}=E_{Q}$ and $\Delta_{Q}^{j}=\Delta_{Q}, j \in\{1,2\}$. 
By a local $T 1$ Theorem (see e.g. [1, 13, 25]), it suffices to show that

$$
|\langle T f, g\rangle| \lesssim\left(1+\mathbf{T}_{\text {loc }}\right)\left|Q_{0}\right|,
$$

where $f$ and $g$ are measurable functions with the property $|f|=|g|=\mathbf{1}_{Q_{0}}$. By Lemma 3.5. the expansion of the bilinear form is

$$
\langle T f, g\rangle=\left\langle T E_{Q_{0}} f, g\right\rangle+\left\langle T \sum_{P: P \subset Q_{0}} \Delta_{P} f, E_{Q_{0}} g\right\rangle+\sum_{P, Q: P \cup Q \subset Q_{0}}\left\langle T \Delta_{P} f, \Delta_{Q} g\right\rangle .
$$

Using the assumptions, it is straightforward to verify that

$$
\left|\left\langle T E_{Q_{0}} f, g\right\rangle\right| \lesssim \mathbf{T}_{\text {loc }}\left|Q_{0}\right|^{1 / p_{2}^{\prime}}\left|Q_{0}\right|^{1 / p_{2}}
$$

and, by also using Lemma 3.5. that

$$
\left|\left\langle T \sum_{P: P \subset Q_{0}} \Delta_{P} f, E_{Q_{0}} g\right\rangle\right| \lesssim \mathbf{A} \mathbf{T}_{\text {loc }}\left|Q_{0}\right|^{1 / p_{1}}\left|Q_{0}\right|^{1 / p_{1}^{\prime}}
$$

Since $1 / p_{1}+1 / p_{1}^{\prime}=1=1 / p_{2}+1 / p_{2}^{\prime}$, we are left with estimating the main term,

$$
\sum_{P, Q}\left\langle T \Delta_{P} f, \Delta_{Q} g\right\rangle=\left\{\sum_{P, Q: \ell P<\ell Q}+\sum_{P, Q: \ell P=\ell Q}+\sum_{P, Q: \ell P>\ell Q}\right\}\left\langle T \Delta_{P} f, \Delta_{Q} g\right\rangle,
$$

where all the summations are restricted to dyadic cubes $P, Q$ contained in $Q_{0}$. As is standard, we will assume that $T$ has kernel $K$ which is perfect and that $K(x, y)$ is identically zero for $|x-y|$ sufficiently small. In particular, the sum above can be taken over a finite collection of $P, Q$. We will rearrange the sum as is convenient for us. We emphasize that all of the subsequent estimates are independent of this truncation.

We will show that the second and third forms above obey the desired estimate, and by duality the same will hold for the first term. To state this otherwise, it suffices to consider the two forms

$$
\begin{gathered}
B_{\text {above }}(f, g):=\sum_{P, Q: P \supsetneq Q}\left\langle T\left(\Delta_{P} f\right), \Delta_{Q} g\right\rangle, \\
B_{=}(f, g):=\sum_{P, Q: P=Q}\left\langle T \Delta_{P} f, \Delta_{Q} g\right\rangle,
\end{gathered}
$$

where the summations are restricted to cubes $P, Q$ that are contained in $Q_{0}$. We will suppress this notationwise also in the sequel, but this fact will be used nevertheless. It is noteworthy that the martingale transform inequality is decisive in estimating both of these terms.

\section{The TERM $B_{\text {above }}$}

We address a bookkeeping issue. For the cube $P$ set

$$
\widetilde{\Delta}_{P} f:=\left\{\sum_{P^{\prime} \in \operatorname{ch}(P) \backslash \mathcal{S}_{1}} \frac{\langle f\rangle_{P^{\prime}}}{\left\langle b_{\pi_{\mathcal{S}_{1}} P^{\prime}}^{1}\right\rangle_{P^{\prime}}} \mathbf{1}_{P^{\prime}}\right\}-\frac{\langle f\rangle_{P}}{\left\langle b_{\pi_{\mathcal{S}_{1} P}}^{1}\right\rangle_{P}} \mathbf{1}_{P}
$$

This is closely related to a half-twisted martingale difference associated with $P$. It suffices to show that for any $S \in \mathcal{S}_{1}$,

$$
\left|\mathbf{1}_{\left\{S \neq Q_{0}\right\}} \cdot\langle f\rangle_{S} \sum_{Q: Q \subset S}\left\langle T b_{S}^{1}, \Delta_{Q} g\right\rangle+\sum_{P: \pi_{\mathcal{S}_{1}} P=S} \sum_{Q \subsetneq P}\left\langle T\left(b_{S}^{1} \widetilde{\Delta}_{P} f\right), \Delta_{Q} g\right\rangle\right| \lesssim \mathbf{T}_{\text {loc }}|S| .
$$


Indeed, the sum on the left hand side over $S \in \mathcal{S}_{1}$ equals $B_{\text {above }}(f, g)$, and the collection $\mathcal{S}_{1}$ is a Carleson sequence of cubes. The terms involving $f$ depend only upon $b_{S}^{1}$, which is a convenience.

The first term is easy to estimate. The twisted martingale differences on $g$ telescope, so that

$$
\sum_{Q: Q \subset S} \Delta_{Q} g=g \mathbf{1}_{S}-\frac{\langle g\rangle_{S}}{\left\langle b_{\pi_{\mathcal{S}_{2}} S}^{2}\right\rangle_{S}} b_{\pi_{\mathcal{S}_{2} S}}^{2} \mathbf{1}_{S} .
$$

The ratio of averages is controlled by construction. By the local $T b$ assumptions and construction,

$$
\left|\left\langle T b_{S}^{1}, g \mathbf{1}_{S}\right\rangle\right|+\left|\left\langle T b_{S}^{1}, b_{\pi_{\mathcal{S}^{2}} S}^{2} \mathbf{1}_{S}\right\rangle\right| \lesssim \mathbf{T}_{\text {loc }}|S| .
$$

For the second term, the twisted martingale transform is the decisive point. For pairs of cubes $Q \subsetneq P$, let $P_{Q}$ denote the child of $P$ that contains $Q$. The properties of $T$ being perfect and $\Delta_{Q} g$ having integral zero allow us to write

$$
\begin{aligned}
\left\langle T\left(b_{S}^{1} \widetilde{\Delta}_{P} f\right), \Delta_{Q} g\right\rangle & =\left\langle T\left(b_{S}^{1} \widetilde{\Delta}_{P} f \cdot \mathbf{1}_{P_{Q}}\right), \Delta_{Q} g\right\rangle \\
& =\left\langle\widetilde{\Delta}_{P} f\right\rangle_{P_{Q}}\left\langle T\left(b_{S}^{1} \mathbf{1}_{P_{Q}}\right), \Delta_{Q} g\right\rangle \\
& =\left\langle\widetilde{\Delta}_{P} f\right\rangle_{P_{Q}}\left\langle T b_{S}^{1}, \Delta_{Q} g\right\rangle .
\end{aligned}
$$

We have first restricted the argument of $T$ to the cube $P_{Q}$, pulled out the constant value of $\widetilde{\Delta}_{P} f$ on that cube, and finally extended the argument of $T$ to the entire cube $S$.

Now, fix $Q \subsetneq S$, and define a constant $\varepsilon_{Q}$ by the formula

$$
\varepsilon_{Q}:=\sum_{\substack{P: P \supsetneq Q \\ \pi_{\mathcal{S}_{1} P=S}}}\left\langle\widetilde{\Delta}_{P} f\right\rangle_{P_{Q}} .
$$

These numbers are bounded by a constant, since the sum is telescoping and equals the difference of two $b$-averages of $f$ (or a single average, in the case of $\pi_{\mathcal{S}_{1}} Q \subsetneq S$ ), which are bounded. We can make a direct appeal to the local $T b$ hypothesis:

$$
\begin{aligned}
\left|\sum_{P: \pi_{\mathcal{S}_{1}} P=S} \sum_{Q \subsetneq P}\left\langle\widetilde{\Delta}_{P} f\right\rangle_{P_{Q}}\left\langle T b_{S}^{1}, \Delta_{Q} g\right\rangle\right| & =\left|\sum_{Q \subsetneq S}\left\langle T b_{S}^{1}, \varepsilon_{Q} \Delta_{Q} g\right\rangle\right| \\
& \leq \mathbf{T}_{\text {loc }}|S|^{1 / p_{2}^{\prime}}\left\|\sum_{Q \subsetneq S} \varepsilon_{Q} \Delta_{Q} g\right\|_{p_{2}} \lesssim \mathbf{T}_{\text {loc }}|S| .
\end{aligned}
$$

The construction and the following argument provide the last inequality.

Indeed, let $S_{2}$ be the $\mathcal{S}_{2}$ parent of $S$, and set $\mathcal{R}_{1}:=\left\{S_{2}\right\}$. Let $\mathcal{R}_{2}$ be the $\mathcal{S}_{2}$ children of $S_{2}$ strictly contained in $S$, and inductively set $\mathcal{R}_{k+1}$ to be the $\mathcal{S}_{2}$ children of cubes $R \in \mathcal{R}_{k}$. Each function below is a twisted martingale transform of $g$ :

$$
\gamma_{R}:=\sum_{\substack{\pi_{\mathcal{S}_{2}} Q=R \\ Q \subsetneq S}} \varepsilon_{Q} \Delta_{Q} g, \quad R \in \bigcup_{k=1}^{\infty} \mathcal{R}_{k} .
$$

There holds $\left\|\gamma_{R}\right\|_{p_{2}} \lesssim|R \cap S|^{1 / p_{2}}$, by the martingale transform inequality, Theorem 2.3. and the fact that $g$ is a bounded function. Moreover, from (3.1), it follows that

$$
\sum_{R \in \mathcal{R}_{k}}|R \cap S| \lesssim \tau^{k}|S|
$$


where $0<\tau<1$ is fixed. Hence,

$$
\begin{aligned}
\left\|\sum_{Q \subsetneq S} \varepsilon_{Q} \Delta_{Q} g\right\|_{p_{2}}^{p_{2}} & =\left\|\sum_{k=1}^{\infty} \sum_{R \in \mathcal{R}_{k}} k^{-1+1} \gamma_{R}\right\|_{p_{2}}^{p_{2}} \\
& \lesssim \sum_{k=1}^{\infty} k^{p_{2}} \sum_{R \in \mathcal{R}_{k}}\left\|\gamma_{R}\right\|_{p_{2}}^{p_{2}} \\
& \lesssim|S| \sum_{k=1}^{\infty} k^{p_{2}} \tau^{k} \lesssim|S| .
\end{aligned}
$$

This completes the analysis of the above form.

\section{THE DIAGONAL TERM}

The diagonal term is frequently the more delicate part of these arguments. Also, one can compare this argument to that of [3. Section 8.1]. Before beginning the main thrust of the argument, a particular consequence of the martingale transform inequality is needed. Set for $j=1,2$,

$$
\square_{Q}^{j} h:=\left|D_{Q}^{j} h\right|+ \begin{cases}\mathbf{1}_{Q} & \text { a child of } Q \text { is in } \mathcal{S}_{j}, \\ 0 & \text { otherwise, }\end{cases}
$$

where $D_{Q}^{j} h$ is the half-twisted martingale difference, defined in (2.5), with $\mathcal{S}_{0}^{j}:=$ $\pi_{\mathcal{S}_{j}} Q$, terminal cubes $\mathcal{T}^{j}:=\operatorname{ch}_{\mathcal{S}_{j}}\left(S_{0}^{j}\right)$, and function $b^{j}:=b_{S_{0}^{j}}^{j}$. More explicitly,

$$
D_{Q}^{j} h=\sum_{Q^{\prime} \in \operatorname{ch}(Q) \backslash \mathcal{S}_{j}}\left\{\frac{\langle h\rangle_{Q^{\prime}}}{\left\langle b_{\left.\pi_{\mathcal{S}_{j} Q^{\prime}}^{j}\right\rangle_{Q^{\prime}}}\right.}-\frac{\langle h\rangle_{Q}}{\left\langle b_{\pi_{\mathcal{S}_{j} Q}}^{j}\right\rangle_{Q}}\right\} \mathbf{1}_{Q^{\prime}}
$$

Roughly speaking, the second summand in (5.1) should be thought of as accounting for the missing terminal cubes in the definition of $D_{Q}^{j}$ - it is even slightly larger than required for that purpose.

We have an inequality

$$
\left\|\left[\sum_{Q: Q \subset Q_{0}}\left(\square_{Q}^{1} f\right)^{2}\right]^{1 / 2}\right\|_{q} \lesssim\left|Q_{0}\right|^{1 / q}, \quad 1<q<\infty .
$$

The corresponding inequality holds also for $g$. Indeed, by first introducing independent Rademacher variables $\left\{\epsilon_{Q}\right\}_{\left\{Q \subset Q_{0}\right\}}$ that are jointly supported on a probability space $\Omega=\{-1,1\}^{\left\{Q \subset Q_{0}\right\}}$ and then using Khintchine's inequality,

$$
\left\|\left[\sum_{Q: Q \subset Q_{0}}\left|D_{Q}^{1} f\right|^{2}\right]^{1 / 2}\right\|_{q} \lesssim\left[\int_{\mathbf{R}^{n}} \int_{\Omega}\left|\sum_{Q: Q \subset Q_{0}} \epsilon_{Q} D_{Q}^{1} f\right|^{q} d \epsilon d x\right]^{1 / q} .
$$

We proceed as at the end of Section 4, using the half-twisted inequality of Theorem [2.6. This yields an upper bound $c\left|Q_{0}\right|^{1 / q}$ for the last term. To estimate the remaining part of (5.2), we first apply the Fefferman-Stein maximal inequality to reduce to the $L^{q}$-norm of the square function $\left\{\sum_{S \in \mathcal{S}_{1}} \mathbf{1}_{S}\right\}^{1 / 2}$. The proof of inequality (5.2) is finished by proceeding as above.

To control the diagonal term, it therefore suffices to show that

$$
\left|\left\langle T \Delta_{Q} f, \Delta_{Q} g\right\rangle\right| \lesssim\left(1+\mathbf{T}_{\mathrm{loc}}\right) \sum_{Q^{1}, Q^{2} \in \operatorname{ch}(Q)}\left\langle\square_{Q}^{1} f\right\rangle_{Q^{1}}|Q|\left\langle\square_{Q}^{2} g\right\rangle_{Q^{2}}, \quad Q \subset Q_{0} .
$$


For cube $Q$ and child $Q^{1}$ of $Q,\left(\Delta_{Q} f\right) \mathbf{1}_{Q^{1}}$ is either a constant multiple of $b_{\pi_{\mathcal{S}_{1}} Q^{1}}^{1} \mathbf{1}_{Q^{1}}$ or, in the exclusive case that $Q^{1}$ is also a stopping cube, a linear combination of this function and $b_{Q^{1}}^{1}$. In both cases, the coefficients in the linear combination are dominated by $c\left\langle\square_{Q}^{1} f\right\rangle_{Q^{1}}$. Therefore, inequality (5.3) follows from this lemma.

Lemma 5.4. Suppose that $Q \subset Q_{0}$. Then, if $Q^{j}$ is a child of $Q$ and $b^{j} \in$ $\left\{b_{\pi_{\mathcal{S}_{j} Q}^{j}}, b_{Q^{j}}^{j}\right\}$ with $j=1,2$,

$$
\left|\left\langle T\left(b^{1} \mathbf{1}_{Q^{1}}\right), b^{2} \mathbf{1}_{Q^{2}}\right\rangle\right| \lesssim\left(1+\mathbf{T}_{\text {loc }}\right)|Q| .
$$

Proof. Let us consider the case $b^{j}=b_{\pi_{\mathcal{S}_{j} Q}}^{j}, j=1,2$. The other cases are similar but easier. Suppose first that $Q^{1} \neq Q^{2}$. Then, since $T$ is perfect, we see that $K$ is constant on $Q^{2} \times Q^{1}$. Hence, by denoting the midpoint of $Q^{j}$ by $x_{Q^{j}}$,

$$
\begin{aligned}
\left|\left\langle T\left(b^{1} \mathbf{1}_{Q^{1}}\right), b^{2} \mathbf{1}_{Q^{2}}\right\rangle\right| & =\left|K\left(x_{Q^{2}}, x_{Q^{1}}\right)\right| \cdot\left|\int_{Q^{1}} b_{\pi_{\mathcal{S}_{1} Q}}^{1}(y) d y\right| \cdot\left|\int_{Q^{2}} b_{\pi_{\mathcal{S}_{2}}}^{2}(x) d x\right| \\
& \lesssim|Q| .
\end{aligned}
$$

In the last step we used the kernel size estimate.

Then we suppose that $Q^{1}=Q^{2}$. We let $b_{Q^{1}}^{2}$ be the $p_{2}$-accretive function, associated with the cube $Q^{1}$. It suffices to estimate the following terms:

$$
\left|\left\langle T\left(b^{1} \mathbf{1}_{Q^{1}}\right),\left\langle b^{2}\right\rangle_{Q^{1}} b_{Q^{1}}^{2}\right\rangle\right|+\left|\left\langle T\left(b^{1} \mathbf{1}_{Q^{1}}\right), b^{2} \mathbf{1}_{Q^{1}}-\left\langle b^{2}\right\rangle_{Q^{1}} b_{Q^{1}}^{2}\right\rangle\right| .
$$

The first term is bounded by

$$
\left|\left\langle b^{2}\right\rangle_{Q^{1}}\right| \cdot\left|\left\langle b^{1} \mathbf{1}_{Q^{1}}, T^{*}\left(b_{Q^{1}}^{2}\right)\right\rangle\right| \lesssim \mathbf{T}_{\text {loc }}|Q|
$$

Here we used Hölder's inequality, with both exponents $p_{2}$ and $p_{1}$. To estimate the second term in (5.5), the crucial step is to remove the characteristic function $\mathbf{1}_{Q^{1}}$ from within $T\left(b^{1} \mathbf{1}_{Q^{1}}\right)$. For this purpose, let us observe that the function $B^{2}:=$ $b^{2} \mathbf{1}_{Q^{1}}-\left\langle b^{2}\right\rangle_{Q^{1}} b_{Q^{1}}^{2}$ is supported on $Q^{1}$ and has zero integral. By the assumption that $T$ is perfect,

$$
\left|\left\langle T\left(b^{1} \mathbf{1}_{Q^{1}}\right), B^{2}\right\rangle\right|=\left|\left\langle T b^{1}, B^{2}\right\rangle\right| \lesssim \mathbf{T}_{\text {loc }}|Q| .
$$

In the last step, we split the dual form into two other forms and use Hölder's inequality, with exponent $p_{2}$, for the individual forms separately.

\section{REFERENCES}

[1] P. Auscher, S. Hofmann, C. Muscalu, T. Tao, and C. Thiele, Carleson measures, trees, extrapolation, and $T(b)$ theorems, Publ. Mat. 46 (2002), no. 2, 257-325, DOI 10.5565/PUBLMAT_46202_01. MR.1934198 (2003f:42019)

[2] Pascal Auscher, Steve Hofmann, Michael Lacey, Alan McIntosh, and Ph. Tchamitchian, The solution of the Kato square root problem for second order elliptic operators on $\mathbb{R}^{n}$, Ann. of Math. (2) 156 (2002), no. 2, 633-654, DOI 10.2307/3597201. MR1933726 (2004c:47096c)

[3] P. Auscher and E. Routin, Local Tb theorems and Hardy inequalities, J. Geometric Anal. 23 (2013), no. 1, 303-374. MR3010282

[4] Pascal Auscher and Qi Xiang Yang, BCR algorithm and the T(b) theorem, Publ. Mat. 53 (2009), no. 1, 179-196, DOI 10.5565/PUBLMAT_53109_08. MR2474120 (2010i:42021)

[5] G. Beylkin, R. Coifman, and V. Rokhlin, Fast wavelet transforms and numerical algorithms. I, Comm. Pure Appl. Math. 44 (1991), no. 2, 141-183, DOI 10.1002/cpa.3160440202. MR.1085827(92c:65061)

[6] D. L. Burkholder, Boundary value problems and sharp inequalities for martingale transforms, Ann. Probab. 12 (1984), no. 3, 647-702. MR744226 (86b:60080) 
[7] Donald L. Burkholder, Explorations in martingale theory and its applications, École d'Été de Probabilités de Saint-Flour XIX-1989, Lecture Notes in Math., vol. 1464, Springer, Berlin, 1991, pp. 1-66, DOI 10.1007/BFb0085167. MR1108183 (92m:60037)

[8] Michael Christ, A T (b) theorem with remarks on analytic capacity and the Cauchy integral, Colloq. Math. 60/61 (1990), no. 2, 601-628. MR.1096400 (92k:42020)

[9] R. R. Coifman, Peter W. Jones, and Stephen Semmes, Two elementary proofs of the $L^{2}$ boundedness of Cauchy integrals on Lipschitz curves, J. Amer. Math. Soc. 2 (1989), no. 3, 553-564, DOI 10.2307/1990943. MR986825 (90k:42017)

[10] Guy David and Jean-Lin Journé, A boundedness criterion for generalized Calderón-Zygmund operators, Ann. of Math. (2) 120 (1984), no. 2, 371-397, DOI 10.2307/2006946. MR763911 (85k:42041)

[11] Tadeusz Figiel, Singular integral operators: a martingale approach, Geometry of Banach spaces (Strobl, 1989), London Math. Soc. Lecture Note Ser., vol. 158, Cambridge Univ. Press, Cambridge, 1990, pp. 95-110. MR.1110189 (94e:42015)

[12] Steve Hofmann, Michael Lacey, and Alan McIntosh, The solution of the Kato problem for divergence form elliptic operators with Gaussian heat kernel bounds, Ann. of Math. (2) 156 (2002), no. 2, 623-631, DOI 10.2307/3597200. MR.1933725 (2004c:47096b)

[13] Steve Hofmann, Local T(b) theorems and applications in PDE, Harmonic analysis and partial differential equations, Contemp. Math., vol. 505, Amer. Math. Soc., Providence, RI, 2010, pp. 29-52, DOI 10.1090/conm/505/09914. MR2664559(2011e:42024)

[14] Steve Hofmann, Local Tb theorems and applications in PDE, International Congress of Mathematicians. Vol. II, Eur. Math. Soc., Zürich, 2006, pp. 1375-1392. MR2275650 (2007k:42030)

[15] Tuomas Hytönen and Henri Martikainen, Non-homogeneous Tb theorem and random dyadic cubes on metric measure spaces, J. Geom. Anal. 22 (2012), no. 4, 1071-1107. MR2965363

[16] Tuomas Hytönen and Henri Martikainen, On general local Tb theorems, Trans. Amer. Math. Soc. 364 (2012), no. 9, 4819-4846, DOI 10.1090/S0002-9947-2012-05599-1. MR2922611

[17] Tuomas Hytönen and Fedor Nazarov, The local Tb theorem with rough test functions, available at http://www.arxiv.org/abs/1206.0907.

[18] Tuomas P. Hytönen and Antti V. Vähäkangas, The local non-homogeneous Tb theorem for vector-valued functions (2012), available at arxiv:1201.0648.

[19] Michael T. Lacey, Stefanie Petermichl, and Maria Carmen Reguera, Sharp $A_{2}$ inequality for Haar shift operators, Math. Ann. 348 (2010), no. 1, 127-141, DOI 10.1007/s00208-009-0473y. MR2657437 (2011i:42027)

[20] Michael T. Lacey, Eric T. Sawyer, and Ignacio Uriarte-Tuero, Astala's conjecture on distortion of Hausdorff measures under quasiconformal maps in the plane, Acta Math. 204 (2010), no. 2, 273-292, DOI 10.1007/s11511-010-0048-5. MR2653055(2011f:30043)

[21] Michael T. Lacey, Eric T. Sawyer, Ignacio Uriarte-Tuero, and Chun-Yen Shen, The two weight inequality for Hilbert transform, coronas, and energy conditions, available at http://www . arxiv.org/abs/1108.2319.

[22] Michael T. Lacey, Eric T. Sawyer, Ignacio Uriarte-Tuero, and Chun-Yen Shen, Two weight inequality for the Hilbert transform: A real variable characterization, available at http:// www. arxiv.org/abs/1201.4319.

[23] Camil Muscalu, Terence Tao, and Christoph Thiele, Multi-linear operators given by singular multipliers, J. Amer. Math. Soc. 15 (2002), no. 2, 469-496, DOI 10.1090/S0894-0347-0100379-4. MR 1887641 (2003b:42017)

[24] Stephanie Anne Salomone, b-weighted dyadic BMO from dyadic BMO and associated $T(b)$ theorems, Collect. Math. 61 (2010), no. 2, 151-171, DOI 10.1007/BF03191239. MR2666228 (2011e:42031)

[25] Elias M. Stein, Harmonic analysis: real-variable methods, orthogonality, and oscillatory integrals, with the assistance of Timothy S. Murphy, Monographs in Harmonic Analysis, III, Princeton Mathematical Series, vol. 43, Princeton University Press, Princeton, NJ, 1993. MR $1232192(95 \mathrm{c}: 42002)$

School of Mathematics, Georgia Institute of Technology, Atlanta, Georgia 30332 E-mail address: lacey@math.gatech.edu

School of Mathematics, Georgia Institute of Technology, Atlanta, Georgia 30332

E-mail address: antti.vahakangas@helsinki.fi 